\title{
Supporting Business Model Modelling: a Compromise between Creativity and Constraints
}

\author{
Boris Fritscher and Yves Pigneur \\ University of Lausanne, 1015 Lausanne, Switzerland, \\ boris.fritscher@unil.ch, yves.pigneur@unil.ch
}

\begin{abstract}
Diagrams and tools help to support task modelling in engineering and process management. Unfortunately they are unfit to help in a business context at a strategic level, because of the flexibility needed for creative thinking and user friendly interactions. We propose a tool which bridges the gap between freedom of actions, encouraging creativity, and constraints, allowing validation and advanced features.
\end{abstract}

\section{Introduction}

Representing information and tasks has gained importance at all levels: UML class diagrams, CAD, business process modelling, GDSS, at nearly every stage there are models to help us cope with the complexity of structuring information. Business information management at a strategic level is not an exception to it, but contrary to the other fields it lacks the visual tools to support them. The problem is in part due to the business objects which have no real fixed representation that can be formalized by a specification, and also due to the freedom needed in such models to allow a creative thinking process. These requirements make it difficult to utilize the more classical task modelling tools which have more strict representation of their objects.

For a tool to support an application in the business context, the challenge is to provide enough specialized functionality to enforce the rules of the methodology (meta-model), without compromising the freedom of creativity. This creativity is the necessary intuition for abstracting the business model to a strategic level out of ongoing activities. From a research methodology standpoint we decided to adopt Hevner et al's design science research framework[1], which focuses on solving a real world problem by applying knowledge to an information system prototype conceived iteratively. Therefore, we focused on resolving the gap between creativity and constraints by creating a new tool. This software has to at the same time, be as flexible as a paper based method, but in addition shares features with computer assisted design programmes. This paper explores the compromises which were required and demonstrate the resulting prototype.

First we introduce the business model canvas we choose to support. Then we review some of the existing models and their support tools. In the third section we present the tool and its iterations. We then discuss some of the early testing which was done. In the last section we look at future iterations of the proposed visual tool. 


\section{Designing Business Models}

\subsection{Business Model Canvas}

For our prototype we choose to implement a tool for a very visual business model canvas called the Business Model Ontology[2]. A business model canvas or ontology (BMO) can be described by looking at a set of nine building blocks. These building blocks were derived from an in-depth literature review of a large number of previous conceptualizations of business models. In this depiction, the business model of a company is a simplified representation of its business logic viewed from a strategic standpoint (i.e. on top of Business Process Modelling). The layout of the nine component has its importance as can be seen in figure 1 .

\begin{tabular}{|c|c|c|c|c|}
\hline \multirow[t]{2}{*}{$\begin{array}{l}\text { PARTNER } \\
\text { NETWORK }\end{array}$} & $\begin{array}{c}\text { KEY } \\
\text { ACTIVITIES }\end{array}$ & \multirow[t]{2}{*}{$\begin{array}{c}\text { VALUE } \\
\text { PROPOSITION }\end{array}$} & $\begin{array}{c}\text { CUSTOMER } \\
\text { RELATIONSHIPS }\end{array}$ & $\begin{array}{l}\text { CUSTOMER } \\
\text { SEGMENTS }\end{array}$ \\
\hline & $\begin{array}{c}\text { KEY } \\
\text { RESOURCES }\end{array}$ & & $\begin{array}{l}\text { DISTRIBUTION } \\
\text { CHANNELS }\end{array}$ & \\
\hline \multicolumn{2}{|c|}{ COSTSTRUCTURE } & & \multicolumn{2}{|c|}{ REVENUE STREAMS } \\
\hline
\end{tabular}

Fig. 1. Business Model Ontology Canvas

Each building block can contain elements instantiating the building block's business logic. For example, Customer Segments could be teenagers, families, young single men. Every element is represented by a sticky note in the real world or a distinctively identifiable component in a digital representation.

At the centre there is the Value Proposition, it describes which customer's problems are solved and why the offer is more valuable than similar products from competitors (product, service). The customer themselves are analysed in Customer Segment, separated into groups to help in identifying their needs, desires and ambitions (singles, families). Distribution Channel illustrates how the customer wants to be reached and by whom he is addressed (Internet, store). 
In addition, Customer Relationships specifies what type of relationship the customer expects and how it is establish and maintained with him (promotion, support, individual or mass). To be able to deliverer the value proposition the business has to have Resources (staff, machines, secret knowledge). And transform theses resources through Key Activities into the final product or service (development, production, secret process). Most of the time a business depends also either for resources or for activities on an external Partner Network (logistics, financial), which can provide better quality or a lower price on non essential components. As any business model would not be complete without financial information the last two building blocks focus on cost and revenue: The Cost Structure which should be aligned to the core ideas of the business model (key resources, key activities) and Revenue Streams which mirrors the value the customers are willing to pay an how they will perform the transaction (one time fee, subscription).

The most interesting feature is the ability to describe the business logic of a company on one page: none of the individual elements of the business model canvas are new to business people. But the simple and yet holistic look at a business on a single page is surprisingly new to most of them.

The current state of the canvas has been reached through a number of iterations in the last eight years, during which over hundred students have applied the canvas to a variety of class projects. Moreover, usage is not limited to academia; since the model is freely available a lot of companies have started using it, as well as consulting firms. Gartner for example used it in an adapted version in one of their publication[3]. Particularly interesting is the fact that over three hundred persons have paid a subscription to be part of a community experience to co-write the current book[4] about the canvas.

\subsection{Example}

To better understand the thinking applied to designing a model, we will describe one possible overview of Skype ${ }^{\mathrm{TM}}$ 's business model. Even if for purpose of explaining we choose to present the nine building blocks in a particular order, this is by no means the order which was used when identifying the elements. Also in figure 2 the colour of the sticky notes has no special meaning. Skype's main value proposition is to offer free VoIP \& video calling. In addition, they offer additional services like for example cheap international calls through Skype out. Their customer segments are mainly global mass customers and they also try to target SMEs. To be able to reach their customers Skype uses the internet to distribute their software, but they also bundle a cdrom of their application with some of Logitech $^{\text {TM }}$ 's webcams. Support of their service is also done through their internet website to allow for mass customization. Since the software and the basic service are offered for free, we show the importance of this fact by adding a sticky note called free to revenue streams. The other real revenues are from their bundle agreements and the Skype out charges. Service usage having been described, we focus on how the value proposition is produced. The resources which 
Skype needs to provide their service is their software and the software developers building it. These resources are used in an activity of software development. In addition, Skype has to maintain an activity of fraud management using for resources their staff and partners. Skype is heavily dependent on their partners since, they do not have their own network infrastructure. Therefore, they need payment providers, telecommunication providers, and naturally hardware vendor. Finally, everything has a cost, but it was decided to feature software development and complaint management as the main cost structures.

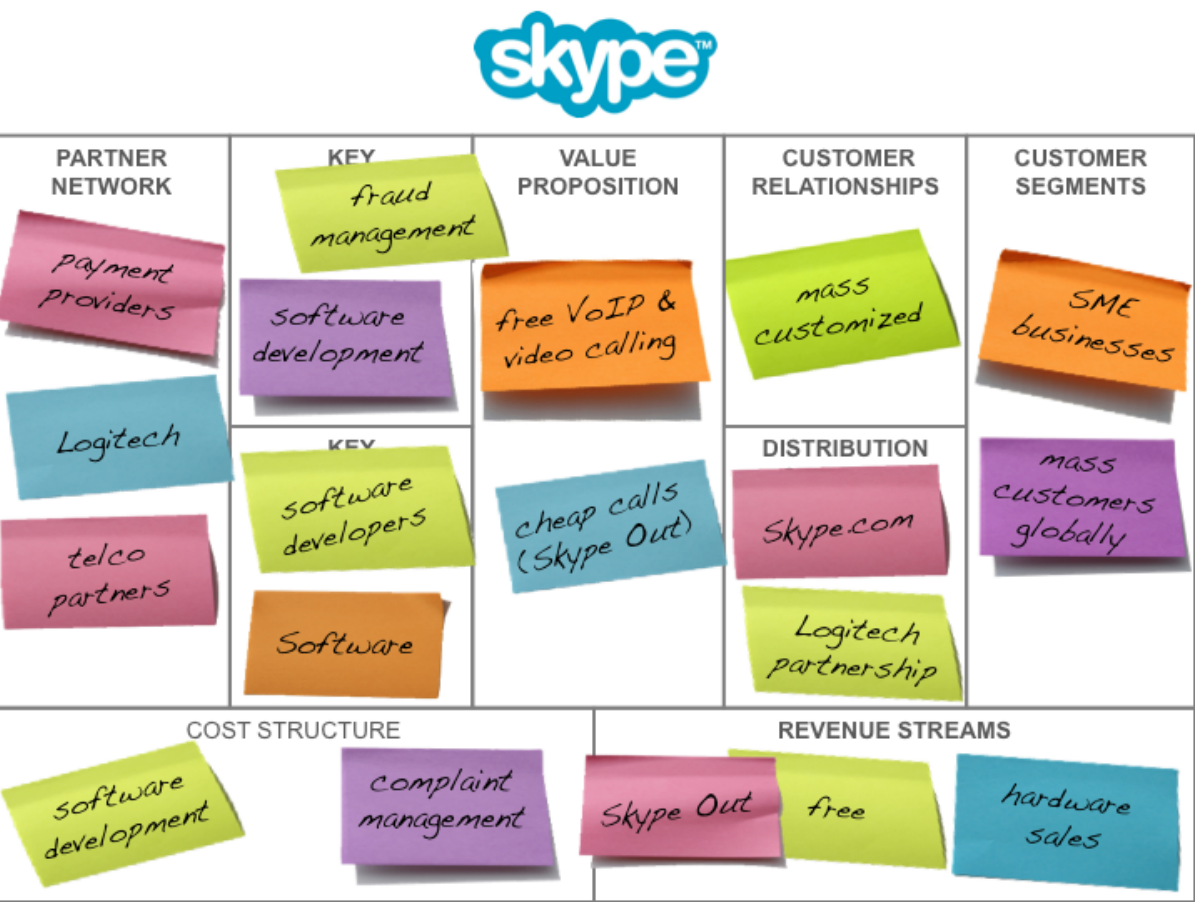

Fig. 2. Skype's Business Model

\subsection{Business Model Ontology}

The canvas shown in figure 1 is a simplified version of the real business model ontology canvas in order to facilitate working with it. The real canvas of the $\mathrm{BMO}$ meta-model is depicted in figure 3 and adds perspectives and links to the building blocks.

When describing the example there already emerges a way to group some building blocks together. We propose to call theses groupings perspectives. As can be seen in figure 3 we identify four perspectives. The value proposition is in 


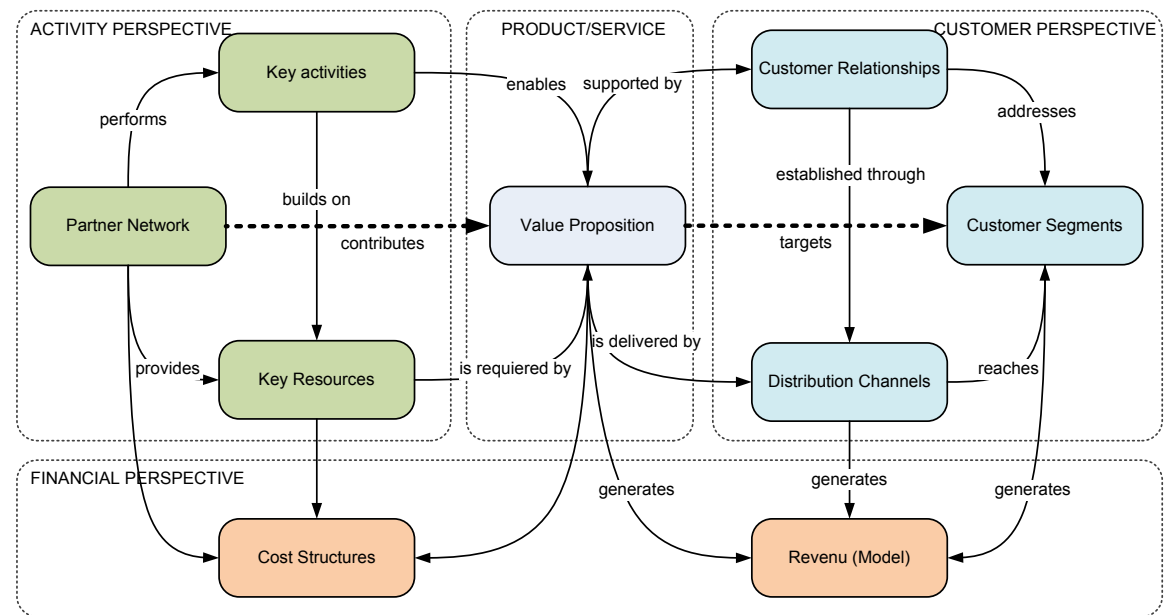

Fig. 3. Business Model Ontology Canvas with the nine building blocks grouped into perspectives and their relations named

its own perspective. Financial aspects like cost and revenue have been grouped into a financial perspective. Moreover, the remaining six blocks have been split into two perspectives: activity and customer. They both are somewhat mirrored, due to the specific position in the canvas. The activity perspective focuses on how the value proposition is produced and the customer perspective how it is consumed.

To imply a strong relationship between two elements there is the possibility to link them. To further understand the meaning a link should convey we named them in figure 3. For example, applied to Skype's canvas: free VoIP targets mass customers, which are reached by skype.com delivering it. This way of connecting the elements can also help in identifying missing elements in neighbouring blocks.

\subsection{Typical Session}

To better understand the BMO methodology let us describe the tasks involved in a paper based session. The power of the method originates from its visual positioning of the block and the relationship they have between each other. Simply adding, removing or changing sticky notes containing a short title, to the building blocks, helps in identifying existing business models, as well as new opportunities. The typical setting is to work on a whiteboard or a big piece of paper, depending on the number of users. The preparation work is to draw an empty canvas on the working surface. After that, the user can start to add an element to any building block, or even temporarily store a sticky note with his idea on a border. As the elements are on sticky notes they can easily be moved, grouped or discarded. If there is a strong relationship between some elements a 
link will be drawn between them. Elements can be grouped together or even be replaced by a more generalized element during the creation process, or elements can be refined and become a new sticky note. After an initial brainstorm, it can be useful to focus on a specific perspective and identify the strong links between elements and see if there are some missing components.

\subsection{Technique and Task}

There are no given task sequences to follow in order to design a business model with the Business Model Canvas[4]. An element (sticky note) can be added at one place and then moved or even removed. The important thing is the discussion it generated, and perhaps the other elements which have been identified through this discussion. Therefore, instead of identifying small task which can be executed in an undefined order or repeated, we instead propose some techniques to help structure the idea generation without imposing a too formal process. These techniques include, but are not limited to: Brainstorming [5], ideation, visual thinking, customer insight, storytelling, scenarios [4].

In general, it is a good practice to generate ideas by adding everything we think of to the canvas. Like in a Brainstorming session, the ideas have to be written down without jugging them. Some persons are more inspired by visuals; this can be addressed by drawing a sketch of an object illustrating the element that has to be added. The drawn object does not need to be a perfect representation of the element, but can also be a metaphor of the activity. A small illustration can communicate a lot more than a single word. Naturally, at some point the visuals will have to be describes by a text clearly sharing its full meaning and the amount of generated elements through creative thinking will have to be synthesised into a coherent working business model. Perspectives, besides helping to group the nine blocks into fewer components, can be interesting starting points. A business model can have its focus centred on the resources (activity perspective), the value proposition itself, the customer or even focus on the financials at the very beginning. Once the canvas is already populated with elements, it can be helpful to see which element is linked to others. This identifies if an element is missing, another way to get more related elements is to use storytelling. Telling a story involving the elements and how they are connected, can not only show missing or unused elements, but helps in communicating the whole business model to outsiders. The business canvas covers different knowledge area about a company; it is therefore natural that the design activity should be performed in groups, discussing the opinions of each other. The task of co-designing is very important, since every participant has to be aware that his vision is not the only one and should be able to take the stakeholder's perspective about the element which is discussed.

\subsection{Life Cycle}

In its current state the business model canvas[4] and the application supporting it consider a completed canvas as the finished product itself. The business model 
components are not transformed to generate a process, but the canvas as a whole provides a map or an overview for the management of the current service offering, or a future offering, they aspire to reach. Even though, the canvas can be used at different stage of a business model lifecycle and could in future work be extend to be used as a reference for implementing appropriate solution in other tools.

\section{Process}

- Mobilize people to generate new business opportunities: in this first phase the business model canvas can help to set a simple common language through its nine blocks, links and layout.

- Understand the current situation: using the above described techniques the canvas helps to regroup the collected information and hints at missing information.

- Design, extending the business model: with the sticky notes and it all in one page format, alternatives can be identified until a best one emerges.

- Implement the chosen business model: the canvas and techniques like story telling help share the vision and therefore facilitated the implementation.

- Manage the current business model: like strategy maps the canvas could help to monitor the current situation.

In its paper form, the final designed business model canvas is shared as a picture of the sticky notes, or for better sharing it is sometimes recreated in a time consuming task on a graphics program. The biggest drawback of these representations is that they lack any additional semantic value. In the next section we take a look at other visual methodologies and how their tools have tried to overcome this problem.

\section{Overview of Tools Assisting Design}

Most of the time innovation starts on a piece of paper, as a little sketch or some keywords which are written on it. This is done mostly to structure ideas, remember them or help communicate them. Communicating one's ideas can generate discussions and help us generate new ones. In addition, the paper represents the shared knowledge, as well as the history of the collaboration session. Today, there are many techniques to help strengthen the power of manual note taking. Some are best used in a multi-user scenario like Brainstorming[5], while others are intended for a single user like some note taking canvas. The constraints of these techniques can be very structured like in said canvas or unstructured like in Brainstorming which really insist on pushing the boundaries of creativity with its rules. There are also techniques which are semi-structured and can be used as well in a single user, as in a multi-user context, like Mind Maps[6] and concept $\operatorname{maps}[7]$.

Many tools exist to support these techniques, but they are all lacking some features to be really useful in a business modelling context. The more unstructured tools like Mind maps provide a great range of freedom to create elements as 
ideas come to mind, but lack the possibility to impose meta-model constraints. Structured tools, like CAD programs, are for the major part very feature reach, but always geared towards a specific domain. This makes them very powerful for the expert user, but useless for office workers. Furthermore, their complexity often requires a sequential input which hinders creative thinking[8]. For example, Protégé is a powerful ontology editor, but is hard to use by a novice user to simply navigate or add his custom element.

The key is to find the right balance between supporting the model by enforcing its rules and still give the user enough degrees of freedom to allow him to follow his own path of creation. The application should be flexible enough to allow for its interaction to be as semless and close as possible to the paper based version and still enforce the meta-model's constraints to be able to provide additional value.

For example, Consideo-modeler ${ }^{1}$ is a nice solution which implements a complete workflow from strategy level modelling down to process level with simulation possibilities, but it requires having quite a lot of knowledge about their modelling rules and there are advanced dialogs boxes to configure the required attributes. A lot more intuitive, Lombardi's BluePrint ${ }^{2}$ web offering allows for real-time collaborative process modelling design. Their service is a good example of collaborative application done with web technologies, but a process model is a lot more structured than the business model canvas we propose to use. On the other hand a tool like Denim[9] offers great capabilities of zoom levels and ways to create and explore hierarchical content, but through their blank canvas do not provide the block constraint needed to have a meta-model which is stronger than just links. Outpost[10] which has an interesting tangile approach towards sticky notes, suffers from the same problem where the importance is set on the link and not the position. Sketchixml[11] shows how we can forgo the need to create elements before using them by drawing their content directly. Is also proposes direct design feedback through pattern analyse, but is geared towards user interface design.

No tool having all the necessary features of constraint versus freedom we required, we propose our own implementation of a solution meeting our need.

\section{Specification of the Design Artefact}

The initial goal for our prototype was to replicate the sticky note experience of adding, moving and discarding elements in the most natural and intuitive way to users, used to the paper based experience. The goal is not only to mimic the user's interaction experience, but also the way the canvas' possibilities drive the creative session. Thus, keeping the trial and error aspect of paper based sticky notes is a core design choice we made throughout the project.

\footnotetext{
${ }^{1}$ http://www.consideo-modeler.de/

${ }^{2}$ http://www.lombardisoftware.com/
} 


\subsection{Prototypes}

In the current iteration of the prototype, a double click creates a new element and drag and drop moves it (figure 4). Discarding an element is done by replicating a trashcan; drop an element on it to remove it. The intention is to provide a feeling as close as possible to the real world sticky note experience. But the fact that the elements now are digital, allows for new functionalities, like giving elements some attributes which can be used to better describe them. We also added a possibility to store annotations to keep track of design choices or ideas for future changes. Some degrees of freedom of drag and drop operations have been limited to allow only movement between the nine blocks, this to ensure that the meta-model of the canvas is maintained. This and element's attributes gives the virtual canvas a semantic meaning which can contributes to more advanced features.

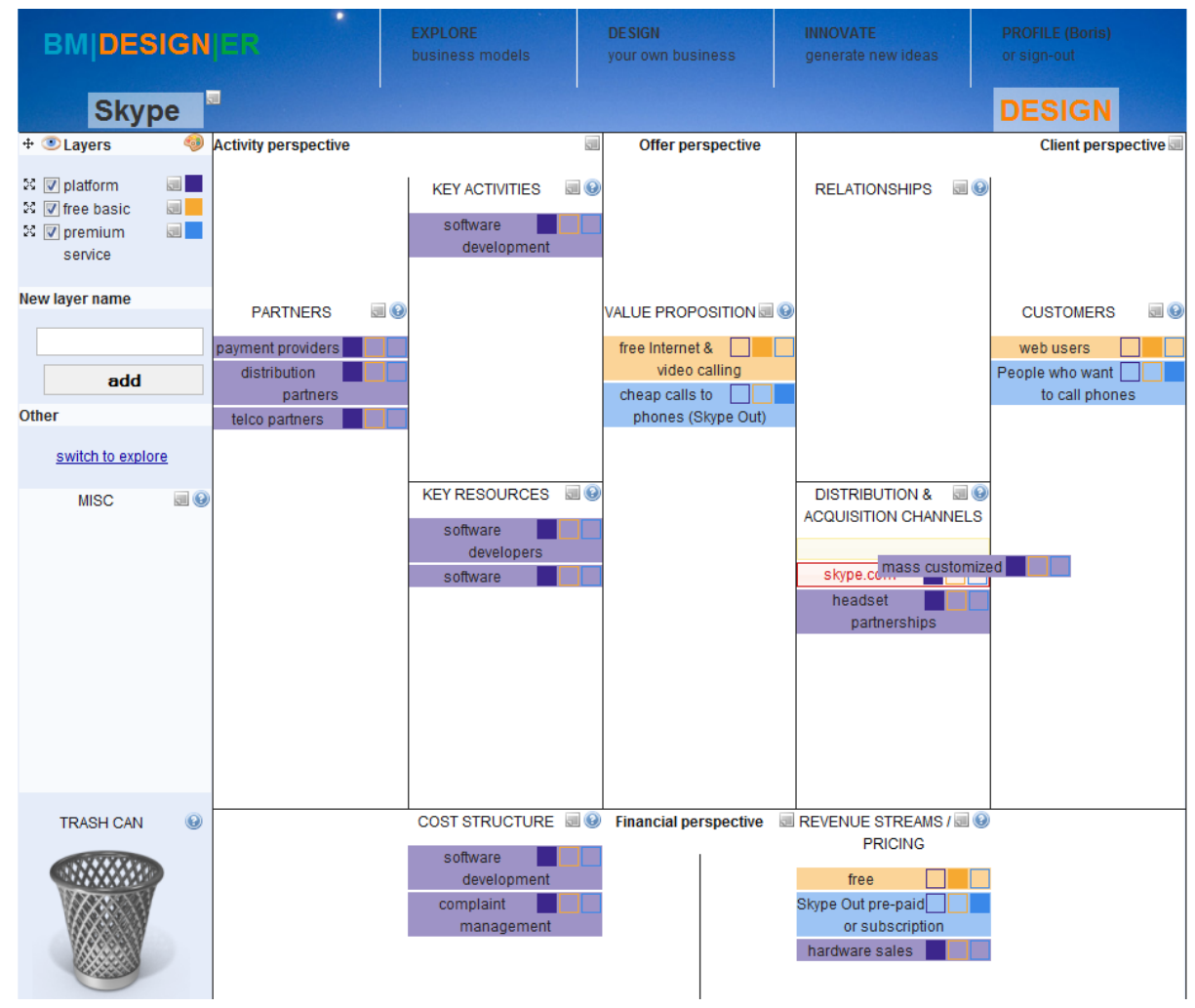

Fig. 4. $B M|D E S I G N| E R$ design view: dragging mass customized from relationships to distribution channels

We have created multiple prototypes and iterations[12], but to a certain level they share all the same set of basic interaction features, as well as the ability 
to link elements (by drag and dropping one element over another). Moreover, a layer feature has also been added. An element can belong to one or more layers and each layer can be turned on or off. For example, a layer identified by a specific colour can be used for each product offering thereby helping to identify which other elements are used by multiple products and thus very important for the business. Different layers can also be used to identify alternatives or future evolutions of the business model.

The concept of versioning has been extended in one of the prototype (figure 5) to allow for taking snapshots (saving its states) and drawing a graph of the history of snapshots of a model. From each snapshot a new branch can be created. This was extended to a notion of merging, by adding multiple instances of a model onto a separate layer of a new empty canvas, thus enabling a limited comparison of the merged business models.

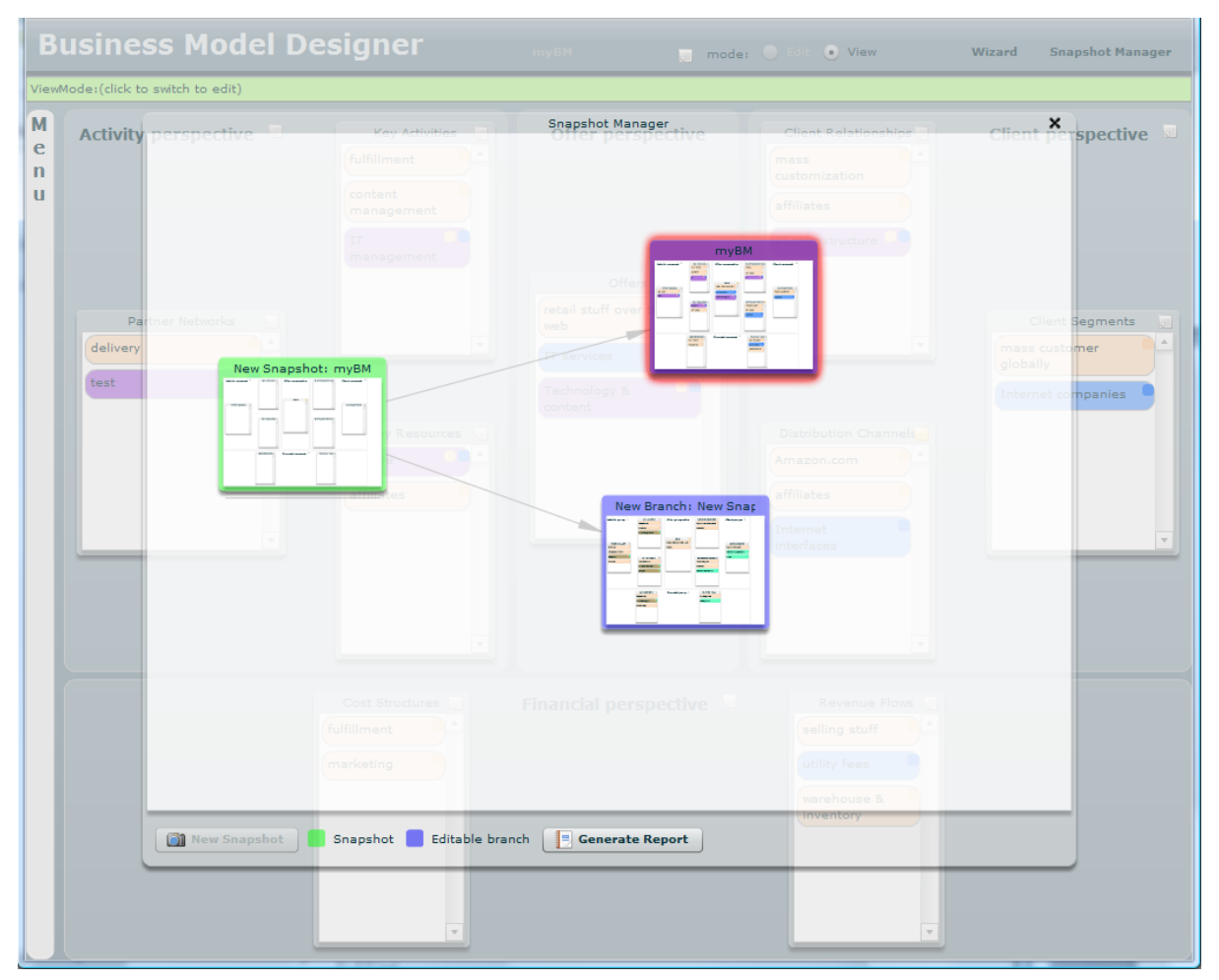

Fig. 5. Flex prototype: overview of snapshots (versions) of a Business Model

To further enhance the usefulness of the digital application we tested the notion of wizard, which would guide the user through some question to help him identify missing elements. This is intended to be used after the initial creative 
thinking process since using it at the beginning would render our tool very structured and thereby missing our initial intention.

The last version of our prototype called $B M|D E S I G N| E R^{3}$ focuses more on ways to navigate through created business models and collaborating on new business model ideas with the help of a community. This is achieved by having a website with modern social web interactions: commenting, voting, tagging, categorizing and searching.

\subsection{Example}

To better illustrate the prototype and its interaction techniques the Skype example was reproduced on $B M|D E S I G N| E R^{4}$ and are described with the help of figures $4,6,7$. When creating a new business model the canvas is empty. By double-clicking one of the nine building blocks the user can add a new element (virtual sticky note) to it through an input dialog box which asks for the element's name. Once it is created it is possible to provide additional attributes. This can be seen in the right hand side of figure 6. Some default attributes are provided for each element type, but new ones can be configured at the user's discretion. Figure 6 also illustrates the input box displayed when clicking an element to rename it (look for the software development element), as well as additional attributes which can be added at the canvas level like tags (top part of the figure). Figure 4 illustrates the drag and drop behaviours. An element can be dragged to a different building block, here mass customized is moved from relationships to distribution channel. While dragging new possible positions for the currently dragged element are highlighted to the user by an empty element at the open spot. The possibility to link two elements is shown when dragging one element over another, the element which is not dragged has its appearance changed (red border and background). Upon releasing an element in a link creation action, the dragged element will go back to its original position, but both elements will have been linked.

In the example shown in figure 4, three layers have been define to make out the free versus premium service Skype offers, and the elements which both these service depend on (platform). Layers are created with the left hand side menu. Adding and removing an element to a layer is done by toggling the corresponding colour swatch on each element. The small sticky note icon which can be seen next to each layer and building block title bring up a dialog to add annotations. Annotations can also have priorities to behave like to-do tasks. In the case of to-do's, the type of annotation shows a number related to its priority. Figure 7 shows annotations of the key activity block from a viewer's angle. The two first figures showed the edition mode (design) of the prototype, while this third one shows the guest mode (explore). In this mode double-clicking an element will draw links it has with other elements. The link between mass customized and skype.com can be seen in figure 7. In view mode the user cannot add new

\footnotetext{
${ }^{3}$ http://bmdesigner.com

${ }^{4}$ http://bmdesigner.com/explore/bm/67/Skype
} 


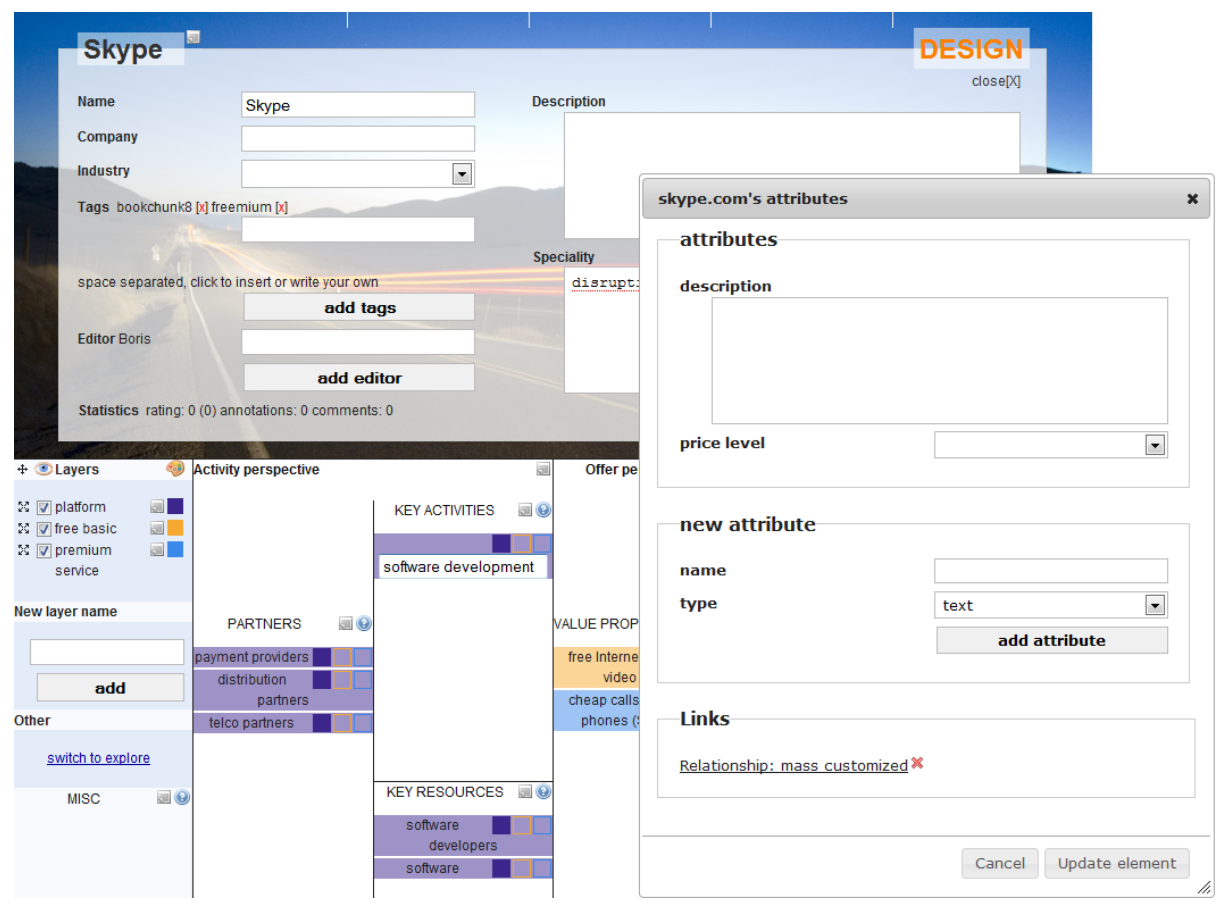

Fig. 6. $B M|D E S I G N| E R$ design view: dialogs to provide additional data

annotations, but he can post comments at the bottom of the canvas, as well as rate it.

\subsection{Implementation}

The prototype is developed on the Grails ${ }^{5}$ framework to capitalize on modern features like convention over coding, Model View Controller and Object Relational Mapping that these web frameworks provide. In addition, Grails has the advantage of being able to use a lot of third party libraries since it run on the java runtime environment. Therefore, by convention the architectzre is separated into data and presentation. The backend data manipulation and persistence is stored in a relational database. The frontend is built in standard web technologies with quiet a few asynchronous JavaScript ${ }^{6}$ calls to be able to provide a seamless interaction without having to reload the web page. In a prior prototype, the same backend technology was used, but the frontend was design in Adobe Flex ${ }^{7}$. This one is mainly used for touch device testing, which still presents challenging in

\footnotetext{
${ }^{5}$ http://grails.org/

${ }^{6}$ http://jquery.com/

${ }^{7}$ http://www.adobe.com/products/flex/
} 


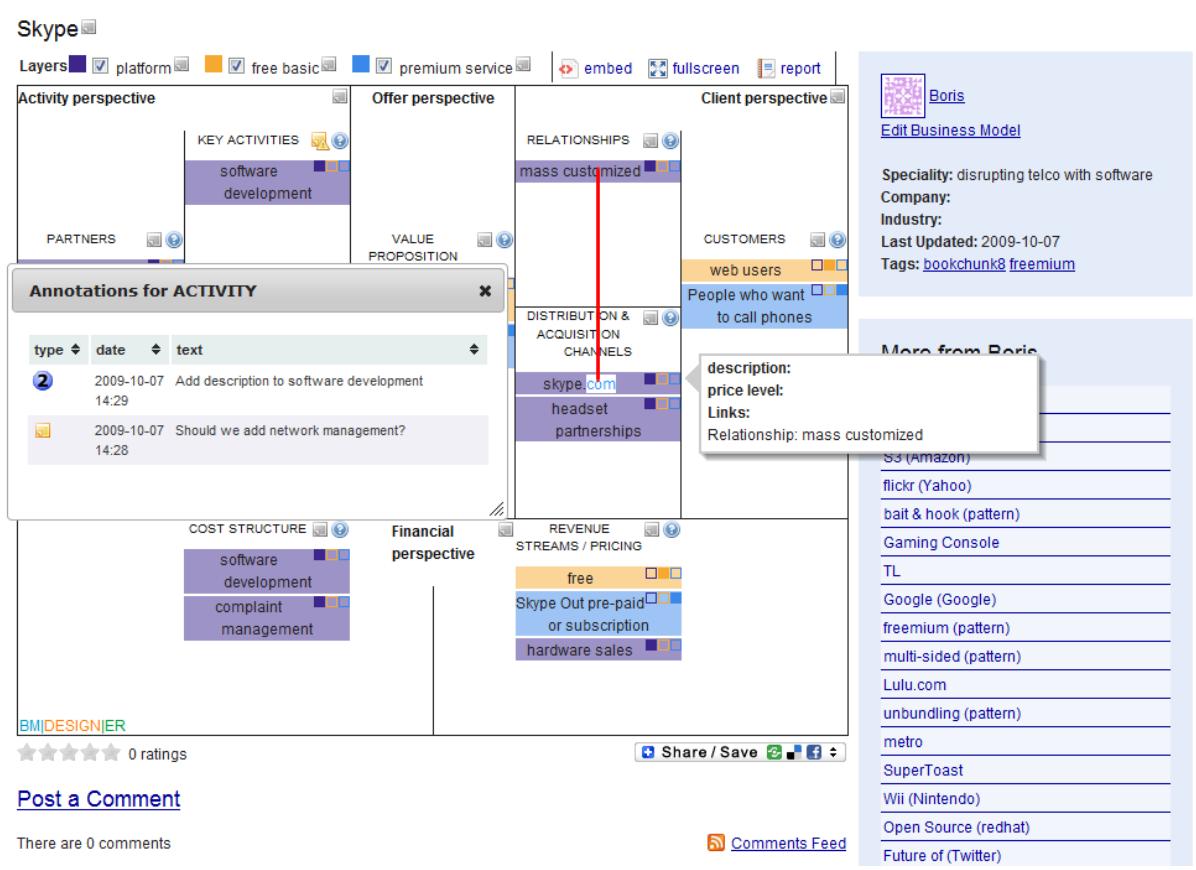

Fig. 7. $B M|D E S I G N| E R$ explore view: displaying links and reading annotations

current web browsers. Other than that web interface are preferred since modifications are simpler, loading time faster and communication with the backend easier.

\section{Evaluation}

Evaluation of the prototype was done using cognitive walkthrough[7] methodology with users having different levels of familiarity of the BMO and coming from different backgrounds (students and businessmen).

Globally feedback was positive. Testers managed to use the prototype without having too much trouble identifying the right action for their intensions. General opinion is that versioning, layers and annotation features may certainly help in providing value over the paper based static solution. There was also a test using an e-Beam ${ }^{8}$ touch screen setup as can be seen in figure 8 .

The idea was twofold: firstly, we wanted to test usability of the tool with a wall projected solution; secondly, we were interested to compare on-screen interaction to the paper based system in relation to group interactions, brainstorming possibilities and other design aspects. We will continue investigating applicability of the tool in a collaborative context, in future iterations, as during preliminary testing mostly usability problems were detected.

\footnotetext{
8 http://e-beam.com/
} 


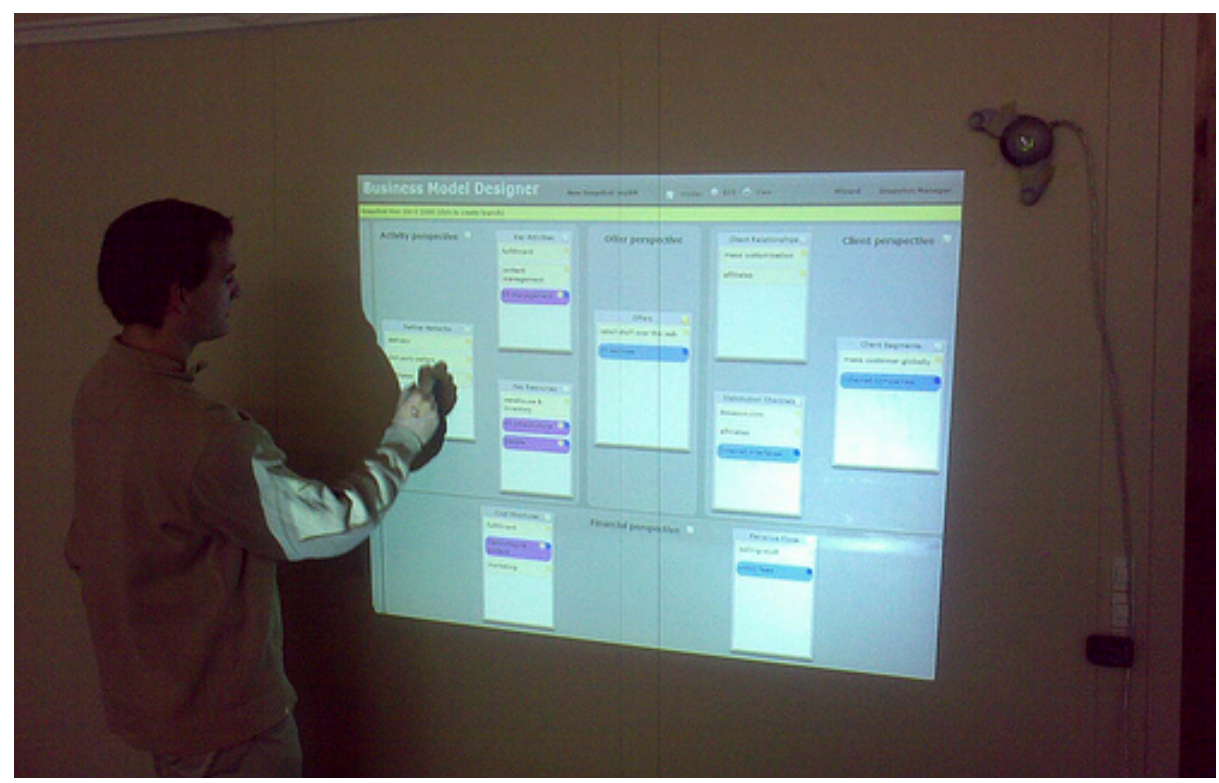

Fig. 8. Prototype projected onto a wall and eBeam interact as pointer

As for the business model community testing site, we just started, but already has over 200 users and 150 models including all the business models from an upcoming book. We hope to be able to study real interaction usage through logs of our tool and use the community as testing platform for new features.

In terms of evaluation, we only are at the beginning and still have a lot of ground to cover. There is an additional difficulty, that in some cases, it is hard to distinguish between problems originating in lack of usability, or in lack of understanding of the business model canvas methodology. For example, do users prefer to use annotations instead of virtual sticky notes because of usability, or methodology? Or even, because on the current prototype all business models are public, due to concerns for the privacy of their model. Either they are generally cautious about their ideas, or this could indicate that even if there are only keywords on the canvas, the users feel it provides enough insight into their activity that it warrants protection. This could be interpreted as an indicatin to the usefulness of the canvas' expressive power.

\section{Discussion and Conclusion}

Our research has been conducted according to Hevner et al's design science research framework[1] and has fulfilled the requirements of his proposed seven guidelines.

1. Design as an Artifact: we developed prototypes which can be evaluated. 
2. Problem Relevance: we have shown that a tool supporting business model innovation is relevant and emerges from a real business need.

3. Design Evaluation: Preliminary evaluation results suggest that the proposed solution is useful in helping to overcome barriers between creativity of business model innovation and constraints of modelling tools.

4. Research Contributions: we contributed to the business model ontology by refining links between the elements.

5. Research Rigor: the business model canvas we based our research on has been validated and is itself based on known ontology research.

6. Design as a Search Process: we iteratively built several prototypes based on evaluation feedback.

7. Communication of Research: Earlier prototypes have been presented in a Master Thesis as well as a workshop on modelling (VMBO 2009, Stockholm).

We have shown that it is possible to find a compromise between freedom and constraints to keep idea generation going, but still enforce a meta-model. Digital alternatives to paper based methodologies can help in providing additional value and style be user friendly enough to be used by office worker.

\subsection{Future Work}

We hope to grow a community around our tool to promote business model innovation at a strategic level and collect valuable feedback. Some tests have been done to investigate collaboration possibility which can be offered by tabletops or touch walls, but this needs further research

There are many possibilities to extend on the prototype as well from an hci perspective as from a business model meta-model one. For example, selective zoom and focus on elements and their details depending on the context, like is done in Denim[9]. We could also imagine moving from a sketch level to a more detailed element view. Testing tangible interactions with real stick notes likes was done in the Outpost project[10] is also an interesting opportunity to further explore ways to enhanced collaboration as well as bridging information and usability. Another interesting direction to explore is to try some kind of SketchiXML[11] application, but instead of drawing UI-elements the user would draw business components and directly generate an appropriate XML or OWL description of the business model. In relation to the stages of the business model canvas' lifecycle, it would also make sense to explore the possibilities offered by multi-fidelity user interfaces[13] to better fit the stage's different needs of flexibility.

As for extending the business model such a tool would benefit of being more than a tool to help designing the model, but also manage the active version by helping at monitoring it like a dashboard. Such a tool could also help in identifing future or alternative version of business model by providing simulation possibilities. 


\section{References}

1. Hevner, A.R., March, S.T., Park, J., Ram, S.: Design science in information systems research. Management Information Systems Quarterly 28(1) (2004) 75-105

2. Osterwalder, A., Pigneur, Y.: An e-business model ontology for modeling ebusiness. In: 15th Bled Electronic Commerce Conference, Bled, Slovenia (2002) 1719

3. Gartner: Getting the right IT: using business models. EXP CIO signature (octobre2007) (2007)

4. Osterwalder, A., Pigneur, Y.: Business Model Generation. businessmodelgeneration.com (2009)

5. Osborn, A.F.: Applied Imagination: Principles and Procedures of Creative Problem-Solving 3rd Edition. 3rd rev edn. Creative Education Foundation (February 1993)

6. Buzan, T.: The mind map book : how to use radiant thinking to maximize your brain's untapped potential. Plume, New York (1993)

7. Novak, J.D., Canas, A.J.: The theory underlying concept maps and how to construct them. University of West Florida (2001)

8. Stacey, M., Eckert, C.: CAD system bias in engineering design. In: Proceedings of the 12th International Conference on Engineering Design. Volume 2. (1999) 923-928

9. Lin, J., Newman, M.W., Hong, J.I., Landay, J.A.: DENIM: finding a tighter fit between tools and practice for web site design. In: Proceedings of the SIGCHI conference on Human factors in computing systems, ACM New York, NY, USA (2000) 510-517

10. Klemmer, S.R., Newman, M.W., Farrell, R., Bilezikjian, M., Landay, J.A.: The designers' outpost: a tangible interface for collaborative web site. In: Proceedings of the 14th annual ACM symposium on User interface software and technology, ACM New York, NY, USA (2001) 1-10

11. Coyette, A., Faulkner, S., Kolp, M., Limbourg, Q., Vanderdonckt, J.: SketchiXML: towards a Multi-Agent design tool for sketching user interfaces based on UsiXML. ACM Press (Proc. of 3rd Int. Workshop on Task Models and Diagrams for user interface design TAMODIA'2004) (2004) 75-82

12. Fritscher, B.: Business Model Designer From Post-it to screen interaction. Ms, University of Lausanne (December 2008)

13. Memmel, T., Vanderdonckt, J., Reiterer, H.: Multi-fidelity user interface specifications. Lecture Notes In Computer Science 5136 (2008) 4357 